\title{
A compact synthetic aperture digital holographic microscope with mechanical movement-free beam scanning and optimized active aberration compensation for isotropic resolution enhancement
}

\author{
YUANBO DENG ${ }^{1}$, CHUNG-HSUAN HAUNG ${ }^{2}$, B. VINOTH ${ }^{2}$, DAPING $\mathrm{CHU}^{1,+}, \mathrm{XIN-JI} \mathrm{LAI}^{2}$, AND CHAU- \\ JERN CHENG ${ }^{2, *}$ \\ ${ }^{1}$ Centre for Photonics Devices and Sensors, Department of Engineering, University of Cambridge, $9 \mathrm{JJ}$ Thomson \\ Avenue, Cambridge, CB3 OFA, UK. \\ ${ }^{2}$ Institute of Electro-Optical Engineering, National Taiwan Normal University, Taipei 11677, Taiwan. \\ *Email: cjcheng@ntnu.edu.tw, +Email: dpc31@cam.ac.uk
}

\begin{abstract}
This study proposes and demonstrates a compact synthetic aperture digital holographic microscope (SA-DHM) with mechanical movement-free beam scanning and optimized active aberration compensation. The SA-DHM system is equipped with a phase-only liquid crystal on silicon (LCOS) device as an active optical element to achieve a compact mechanical movement-free beam steering architecture with aberration correction. A rigorous optimization is conducted to modulate the LCoS device using the designed computer-generated holograms (CGH) to generate a high-quality point spread functions (PSF) for a wide range of scanning angles. An accurate aberration correction is studied with and without oil immersed conditions on microscope objective lens. The performance of the proposed method is analyzed with Siemens star target and the results show its potential capability to achieve isotropic resolution enhancement.
\end{abstract}

\section{Introduction}

Digital holographic microscopy (DHM) is a potential quantitative imaging technique used to study the complex natures such as phase and amplitude of the sample used [1-4]. The conventional DHM system suffers from the limited spatial frequency coverage imposed by the finite numerical aperture of the microscope objective lens used. One method to enhance the frequency coverage is to use synthetic aperture (SA) [5-7]. The synthetic aperture (SA) is achieved by superimposing the holograms of different illumination angles recorded in the spectral domain, so that the spatial frequency coverage can be expanded. The synthesized spectrum can reduce the phase noise of the holographic reconstructed images and enhance its spatial resolution [8-11], but the number of scans required is relatively large, usually several hundred digital holograms are recorded for enhanced resolutions [12]. Separately, the spectrum swelling during frequency overlapping process brings in distortion. Our previous works have introduced an inverse apodization for spectrum normalization to suppress the low-frequency expansion and 
enhance high-frequency components [13-15]. This method enables SA-DHM to increase the spatial resolution by recording only a small number of holograms.

In a SA-DHM setup, a mechanical device, such as a galvo-mirrors, is used to steer the incident beam to provide illumination beam of different angles for scanning the sample. During the scanning, the steering deviation and vibration from the mechanical device will induce phase errors and noises to the holographic recording process, hence decreasing the spatial resolution and signal-to-noise ratio of the reconstruction image. Another issue in SA-DHM is the wavefront aberration originating from the optical system, which also affects the quality and the spatial resolution of holographic reconstructed image. The wavefront compensation of the aberration in digital holography was implemented firstly by the replacement of the actual lens into an interferometry system [16]. Then, a series of aberration correction methods using a numerical reference wavefront in DHM were demonstrated [17-20]. Moreover, a deep learning based aberration compensation method was proposed for fully automatic aberration-free and quantitative phase imaging in DHM [21]. A simple and fast numerical approach was demonstrated for extracting virtual background phase map along with subtracting the reconstructed phase image to implement the compensation of the objective phase curvatures [22]. In addition, the principal component analysis method was applied to decompose the phase map into a set of uncorrelated variables, thus allowing a straightforward compensation of phase aberration without the need of the prior knowledge of the actual setup [23]. Recently, an electrically tunable lens (ETL) was introduced in the reference arm to compensate all the phase distortions for different microscope objectives by controlling precisely the external currents of the ETL to change the reference wavefront, but it requires complex tuning [24] or additional numerical post-processing manipulations [25]. Furthermore, a spiral phase integration method was demonstrated to eliminate the wavefront aberration when measuring confluent samples, however, the acquisition of background area is challenging [26]. There have been several reviews on the theoretical studies of the usefulness of spatial light modulator (SLM) in microscope system [27, 28]. As an extension of these reviews, grating like patterns have been used for the limited angle scanning and aberration correction [29-31]. Several works with wavefront compensation of the PFS were reported under normal illumination conditions [32-34]. However, the scanning spot or the corresponding point spread function (PSF) was formed by a physical lens which brings in aberration.

In this paper, we propose a mechanical-movement-free scanning system using programmable computer-generated holograms (CGH) displayed on a phase-only SLM. Without the use of physical lens, the SLM-based scanning system can generate an array of point spread functions (PSFs) with good quality and shifting linearity at variable focal distances, which is free from the aberration of physical lens and makes the system more 
compact. Meanwhile, the implications of aberration compensation have also been applied to the PSFs to enhance the isotropic resolution of the SA-DHM.

\section{Optimization methodology}

\subsection{Working principle}

In SA-DHM imaging, the image quality is affected by the distortion of point spread function (PSF) as well as the wavefront aberration induced from the different incidence angles of illuminations. The conceptual representation of the proposed system is shown in Fig. 1. As shown in Fig.1, the PSFs with spatial shifts are applied to incident on the condenser lens for generating different angles of plane wave onto the sample. And then the wavefronts are collected by the detection telescopic imaging system along with interference with reference wave to produce digital holograms on the image sensor. The traditional galvo-mirror based mechanical beam steering scheme is replaced by the compact SLM-based beam steering architecture. For the illumination and detection telescopic imaging lens pair, a Zernike polynomials aberration model are applied and iteratively mapped to compensate for the phase aberrations.

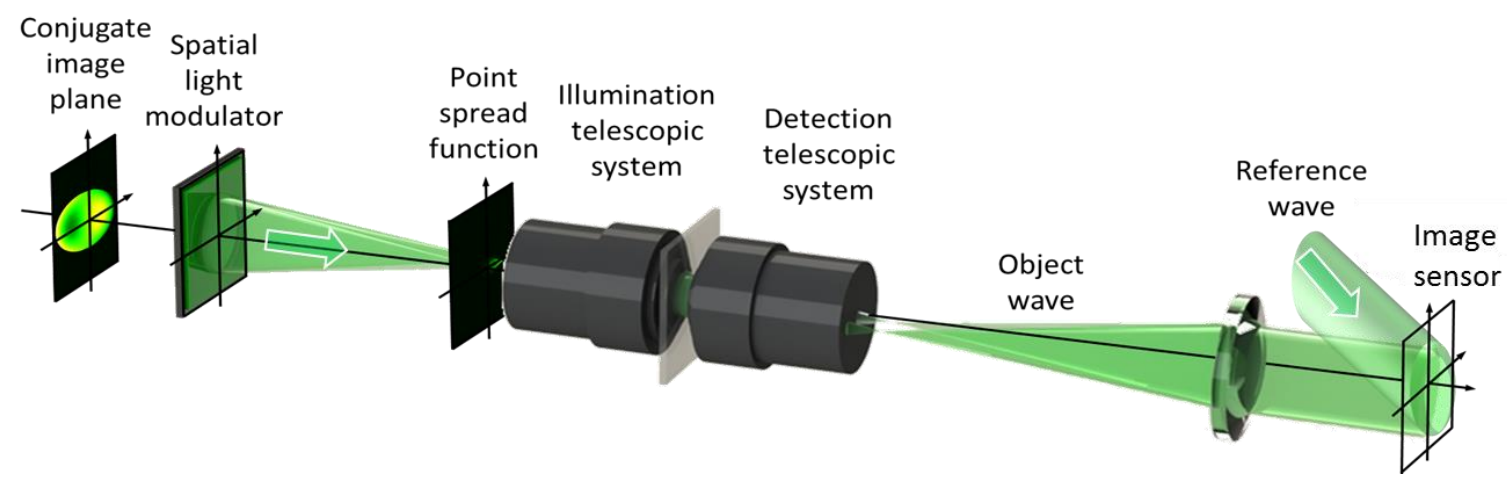

Figure 1. Conceptual representation of compact beam steering with wavefront aberration compensation in synthetic aperture digital holographic microscopy.

A computer-generated hologram (CGH) consists of phase Fresnel lens is designed and displayed on the phase-only liquid crystal on silicon (LCOS) device. The LCoS serves as a SLM to modulate the incident beam to form a focused point at the focal plane of the phase Fresnel lens. A blazed phase grating is then added to the phase Fresnel lens to shift the focused point to achieve a compact beam steering architecture. For a good beam steering mechanism, the scanning PSF should possess three characteristics: small diameter, good intensity profile and no aberration even when the PSF is shifted to a large scanning angle. To realize this, accurate implementation of the phase Fresnel lens and precise phase modulation of the LCoS device are required. Under the assumption that, the generated scanning PSF is of good quality, then we can utilize Zernike polynomial aberration model to compensate for the wavefront aberration from different illumination angles. After the compensation process, uniform illumination 
wavefront at the sample plane can be achieved, which is important for enhancing the spatial resolution of the reconstructed image. The design of $\mathrm{CGH}$ are discussed in the following sections.

\subsection{Design of phase Fresnel lens}

The phase value of the Fresnel lens in $x-y$ plane can be expressed as,

$$
\text { phase value }=\left(\sqrt{x^{2}+y^{2}+z^{2}} \quad z\right) \quad\left(\frac{2}{-}\right)
$$

where $(x, y)$ are the coordinates in the focal plane, $z$ is the focusing distance, and $\lambda$ is the working wavelength. If the calculation area is $x^{2}+y^{2} \ll z^{2}$, then Eq. (1) can be approximated as,

$$
\text { phase value }=\left(\frac{2}{-}\right) \quad\left(\frac{x^{2}+y^{2}}{2 z}\right)
$$

To calculate an appropriate phase Fresnel lens for given conditions, there are two important factors which need to be considered: an appropriate calculation window and a suitable aperture. If the calculation window is not properly set, either insufficient modulation or aliasing would happen. The expression used to generate a Fresnel lens without aliasing $[35,36]$ is given as,

$$
f=\frac{N d x^{2}}{2}
$$

For example, for a green laser $(\lambda=532 \mathrm{~nm})$, if the full effective aperture of the LCoS device consists of $\mathrm{N}=1080$ pixels ( $\mathrm{x}$-axis) with pixel pitch $\mathrm{dx}=6.4 \mu \mathrm{m}$, the minimum focal length is approximated to $83 \mathrm{~mm}$. Focal length shorter than $83 \mathrm{~mm}$ will results in aliasing, or in other word, multiple focused points. On the other side, if the focal length is longer than $83 \mathrm{~mm}$, the phase Fresnel lens will not cover the full information, high frequency part will be lost. Therefore, there is a trade-off between the window calculation and the focal length. An appropriate combination should be carefully considered by satisfying Eq. (3).

When applying the phase Fresnel lens, we also need to consider the masking effect of the CGH displayed on LCOS device $[37,38]$. The aperture shape and profile decides the PSF in the hologram plane. For example, a square aperture and a round aperture will result in a $2 \mathrm{D}$ sinc function and $2 \mathrm{D}$ Bessel function modulated in the reconstruction image, respectively, as shown in Fig. 2. Considering the aperture we need, we use an iris diaphragm to achieve a round aperture. Due to its lever structure with blades, the aperture is a polygon like shape instead of a perfect circular shape, which is shown in Fig. 2(c). For the beam steering operation, the PSF of the system is the resultant scanning spot. In the SA-DHM setup, a physical optical lens will be placed after the scanning PSF 
generated by the phase Fresnel lens, where the scanning spot is located at its front focal plane, as shown in Fig. 1. The scanning PSF serves as a point source for the physical lens to provide a planar illumination for the sample to be observed, and an isotropic intensity profile of the scanning PSF is needed for ideal uniform illumination. The aperture of a conventional LCOS device is rectangular, thus a round aperture is placed after the LCOS to generate an isotropic profile of 2D Bessel function as shown in Fig. 2(d).

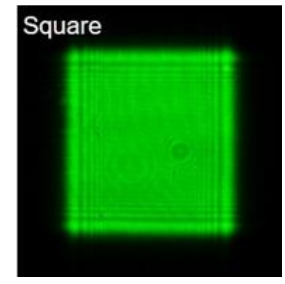

(a)

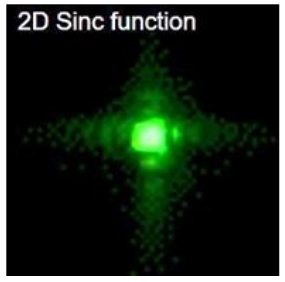

(b)

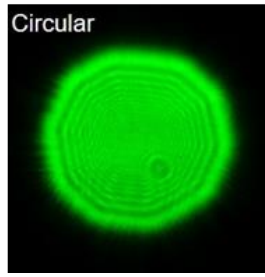

(c)

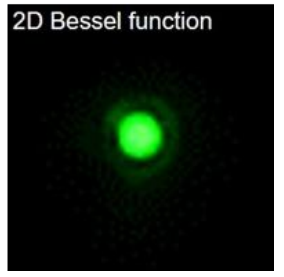

(d)

Figure 2. Aperture mask and its intensity profiles. (a) and (c) shows the square and circular aperture masks similarly (b) and (d) shows its intensity profiles.

\subsection{Optimization of the LCOS device}

To achieve accurate phase modulation, the LCOS device in use needs to be carefully optimized. In general, there are two characteristics needs to be optimized: first, a 3D look-up table compensating for nonlinear phase modulation and liquid crystal cell thickness variation; second, a correct driving voltage range for the working wavelength.

First, due to the nature of liquid crystal, the phase modulation curve for a phase-only LCoS device is not linear, therefore, it is important to measure the phase modulation curve for the working wavelength and find the corresponding voltage value for each phase gray level. For example, to achieve $0-2 \pi$ phase modulation with 256 gray levels, each phase gray level represents $\pi / 128$, then we need to find the accurate corresponding driving voltage to achieve phase modulation from 0 to $2 \pi$ with even phase step of $\pi / 128$. Meanwhile, due to manufacture imperfection, the LCoS device may suffer from thickness variation. If we apply the same voltage to every pixel of the LCoS device, the non-uniform cell thickness will result in phase modulation difference from area to area. The measurement of phase modulation curve and thickness variation [39] can be achieved by measuring the first diffraction order intensity using a set of binary phase gratings on one small area of the LCoS device. Then the first diffraction order intensity is converted to phase modulation value and the modulation curve of this area is obtained. By repeating this measurement on other areas and interpolating the sampled data, we can generate the look-up tables (LUTs) for each gray level. In our experiment, we measure $4 \times 3$ areas on the active LCoS area and interpolate the data to $1920 \times 1080$ LUT for each pixel, and for 0 255, each gray level has its own LUT. Then in total, the LUTs in need can be presented in a $1920 \times 1080 \times 256$ matrix. 
For the LCOS device operated at phase mode, if working under green laser with wavelength of $532 \mathrm{~nm}$, the phase modulation curves for 12 different areas before applying the LUTs are shown in Fig. 3(a) and the curves after applying the LUTs are shown in Fig. $3(b)$. In Fig. $3(a)$, the grating number in $x$ axis represents the binary gratings we use during

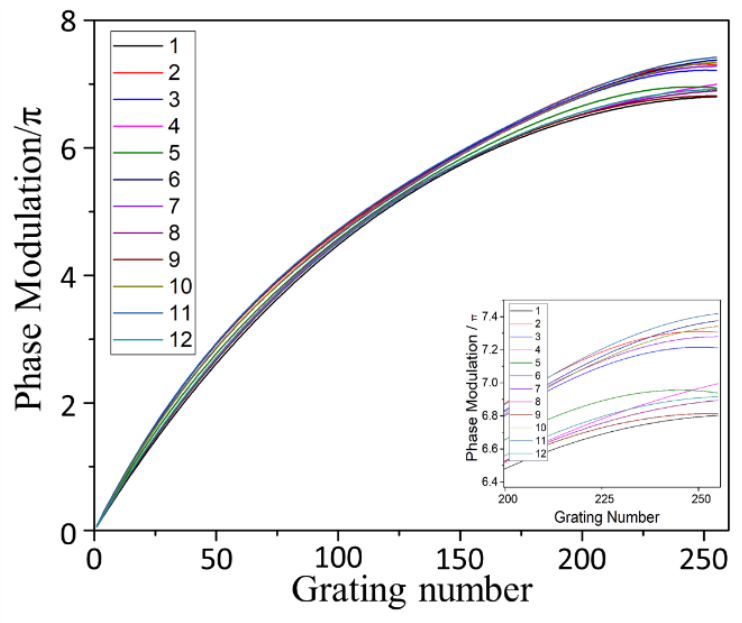

(a)

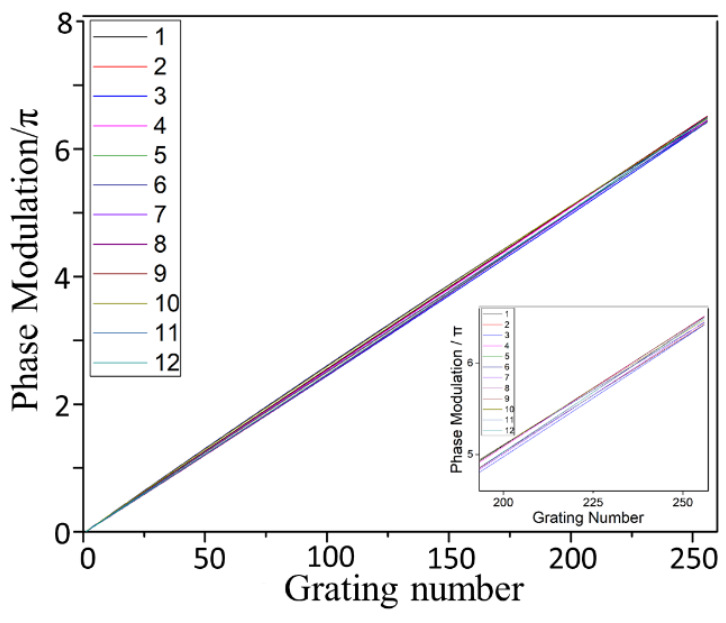

(b)

Figure 3. (a) Phase modulation curves at different areas of the LCoS before calibration; (b) phase modulation curves at different areas of the LCoS device after calibration.

the measurement, the grating number equals the gray levels in hologram. In here, the phase modulation value is in a non-linear relationship with the gray level. Since there is thickness variation, the modulation curves suffer a divergence, where they diverge more significantly when the gray level is large. However, after applying the LUTs, the phase modulation value is in a more linear relationship with the gray level as shown in Fig. 3(b). The divergences between curves also decrease which indicates that the phase-only LCoS device were successfully optimized.

\subsection{Aberration compensation}

As mentioned before, the wavefront correction is based on Zernike polynomials aberration model, which can be found in Table 1. Considering the resultant wavefront distribution is affected by the aberration in the existing optical imaging systems, the wavefront distribution can be simply defined as,

$$
U_{a}(x, y)=P(x, y) e^{j \frac{2}{-W}(x, y)}
$$

where $(x, y)$ is the spatial coordinate, $\mathrm{P}(x, y)$ is the finite aperture size of telescopic imaging lens pair, and $\mathrm{W}(x, y)$ is the wavefront distortion. The PSF of an aberrated coherent imaging system $h_{i}(x, y)$ can be obtained by the Fraunhofer diffraction pattern of the exit wavefront distribution. While the exit wavefront distributions at different 
illumination angles are free from phase distortions, the corresponding PSFs at different angles would be composed by perfect spherical waves and phase gratings. Thus, for implementing the aberration-free from existing phase distortion elements, the wavefront compensated distribution is introduced to the SA-DHM. Then the wavefront propagation through the imaging system, $U_{c}(x, y)$ can be described as follows,

$$
U_{c}(x, y)=h_{d}(x, y) \quad\left[h_{i}(x, y) \quad{ }_{z}(x, y)\right]
$$

Where $\Phi_{z}(x, y)$ is the wavefront compensated for the aberration at the conjugate image plane, which is composed by the Zernike polynomial aberration model $z(x, y)=e^{j \frac{2}{-}\left(\sum_{p=0}^{q} z_{p}\right)}, q$ is the order of Zernike polynomial, the symbol $\otimes$ represents the convolution, $\mathrm{h}_{\mathrm{i}}(x, y)$ and $\mathrm{h}_{\mathrm{d}}(x, y)$ are the PSFs of illumination and detection telescopic imaging lens pair, respectively.

Table 1. The standard Zernike polynomial aberration model representation in Cartesian coordinates.

\begin{tabular}{c|c|c}
\hline Polynomial order & Cartesian coordinates & Description \\
\hline$Z_{0}$ & 1 & Bias \\
$Z_{1}$ & $x$ & Horizontal Tilt \\
$Z_{2}$ & $y$ & Vertical Tilt \\
$Z_{3}$ & $-1+2 x^{2}+2 y^{2}$ & Defocus \\
$Z_{4}$ & $2 x y$ & Oblique Astig \\
$Z_{5}$ & $x^{2}-y^{2}$ & Vertical Astig \\
$Z_{6}$ & $3 x^{3}+3 x y^{2}-2 x$ & Horizontal Coma \\
$Z_{7}$ & $3 y^{3}+3 x^{2} y-2 y$ & Vertical Coma \\
$Z_{8}$ & $6 x^{4}+12 x^{2} y^{2}+6 y^{4}-6 x^{2}-6 y^{2}+1$ & Primary Spherical \\
\hline
\end{tabular}

During the aberration compensation, the conjugated wavefront distribution $\mathrm{U}_{a}(x, y)$ is computed to eliminate the phase distortion. Therefore, the conjugated wavefront of the aberration $\Phi_{z}(x, y)$ is supposedly diffracted to the lensless steering plane and multiplies with phase Fresnel lens plus grating based $\mathrm{CGH}$ to produce the adaptive PSF for aberration-free imaging. Finally, the adaptive PSFs can provide linearly spatial shift onto the illumination telescopic imaging lens to perform the aberration-free in different illumination angles and to extend the spatial frequency coverage for isotropic resolution-enhanced image reconstruction. Thus, the spatial frequency coverage of the SA-DHM system can be indicated as,

$$
U_{o}(\zeta, \eta)=\sum_{m=1}^{M} \sum_{n=1}^{N} \tilde{h}_{d}(u, v) \cdot \tilde{o}(u, v) \cdot\left\{\left[\tilde{h}_{i}(u, v) \cdot \tilde{\Phi}_{z}(u, v)\right] \otimes \delta\left(u+f_{x}^{m}, v+f_{y}^{n}\right)\right\}
$$

where $\tilde{h}_{i}$ and $\tilde{h}_{d}$ is the coherent transfer function of illumination and detection telescopic imaging lens pair in the spatial frequency domain $(u, v)$, the symbols $m$ and $n$ are the number of passbands for synthesis, the $M$ and $N$ are the number of acquisition 
for digital hologram recording and the $f_{x, y}=\sin \theta_{s} / \lambda$ denotes the spatial cut-off frequency of optical system at normal incidence and $\theta_{s}$ is the illumination angle.

A spoken target with 40 line pairs along with optical diffraction limited condition is simulated and the aberration with bias, defocus, Astigmatism and Coma in vertical are shown in Fig. 4(a)-4(d). The first row in Fig. 4 indicates the spatial distribution of wavefront aberration and the second row are the PSF of each aberration. Then, visualized optical diffraction limited images are showed in the third row of Fig. 4 . The final row shows the cross-sectional profiles of the spoken target at 2 line pairs/ $\mu \mathrm{m}$. In Fig. 4(b), the defocus aberration generates reversal contrast on the spoken target, and the vertical astigmatism in Fig. 4(c) generates asymmetric lateral resolution on horizontal and vertical direction, while the vertical coma generates vertical shift on the resultant image as shown in Fig. 4(d).

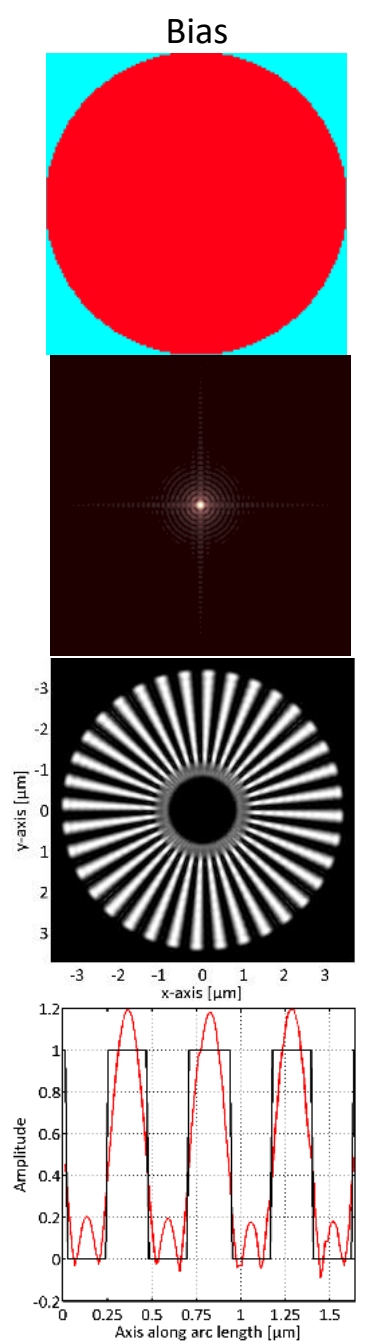

(a)



(b)

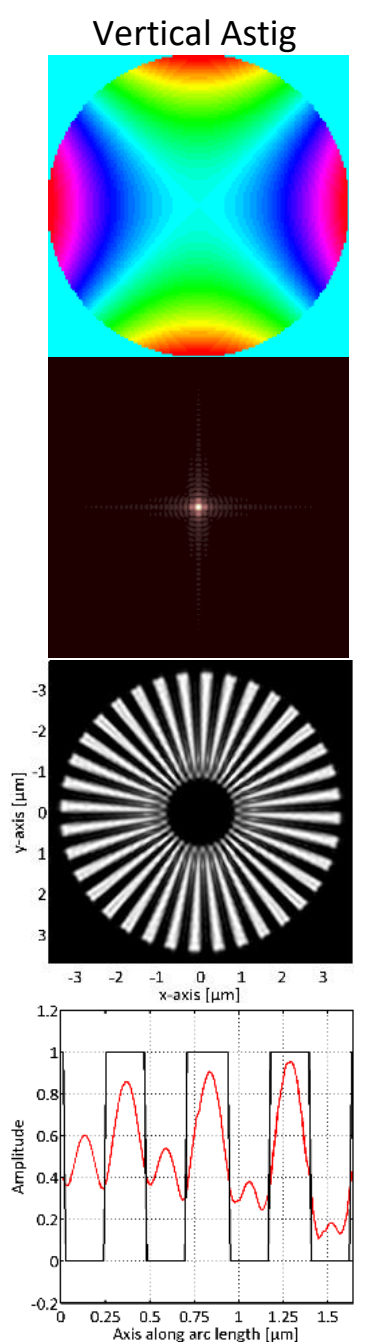

(c)

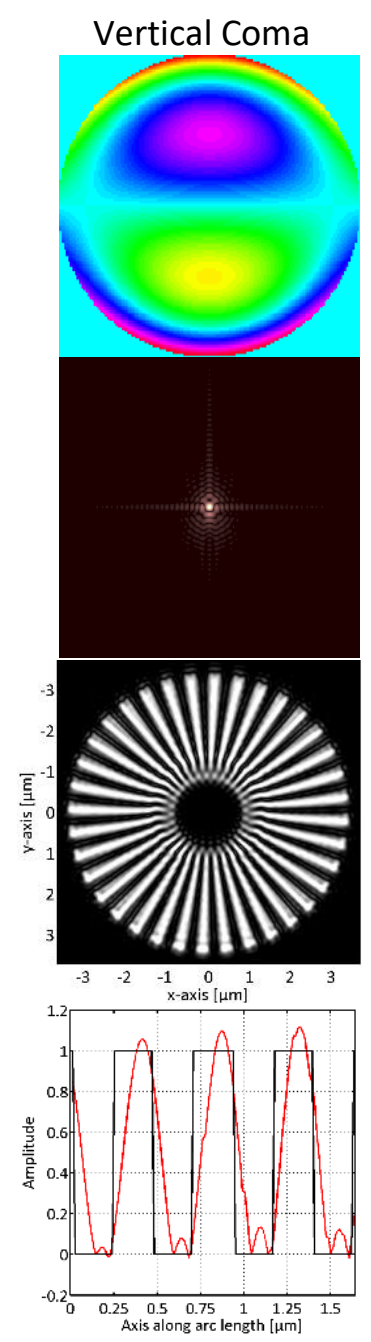

(d)

Figure 4. The 1st row to the 4th row are spatial distribution of wavefront aberration, PSF of each wave aberration, visualized optical diffraction limited image and cross section profile of the spoken target (black 
line indicated the ideal imaging profile, and the red line indicated aberration affected imaging profile at 2 line pairs $/ \mu \mathrm{m}$ ) respectively for (a) bias, (b) defocus, (c) vertical astigmatism and (d) vertical coma.

\section{Experiments and Analysis}

\subsection{Compact SLM-based beam steering architecture}

At first, phase Fresnel lens CGHs are designed and displayed on the LCoS device. A 532 $\mathrm{nm}$ DPSS laser is condensed and then collimated to provide uniform incident beam on the LCoS. Before the LCOS device, a circular aperture is placed, and after the LCoS, a complementary metal-oxide-semiconductor (CMOS) image sensor is used to capture the holographic reconstructed PSF at the distance corresponding to the focal length. Phase Fresnel lens CGHs with different focal lengths have been generated to investigate the relationship between focal length and spot quality. The LCoS device in use is a phase only SLM with pixel size of $6.4 \mu \mathrm{m}$ and pixel numbers of $1920 \times 1080$. The diameter of the circular aperture in our system is $4.26 \mathrm{~mm}$. The scanning spots at different focal lengths from $100 \mathrm{~mm}$ to $400 \mathrm{~mm}$ have been analyzed, the focused PSFs are captured and shown in Fig. 5. The PSFs generated by the phase Fresnel lens stay in good profiles, and as the focal length increases, the size of the PSF increases as well.

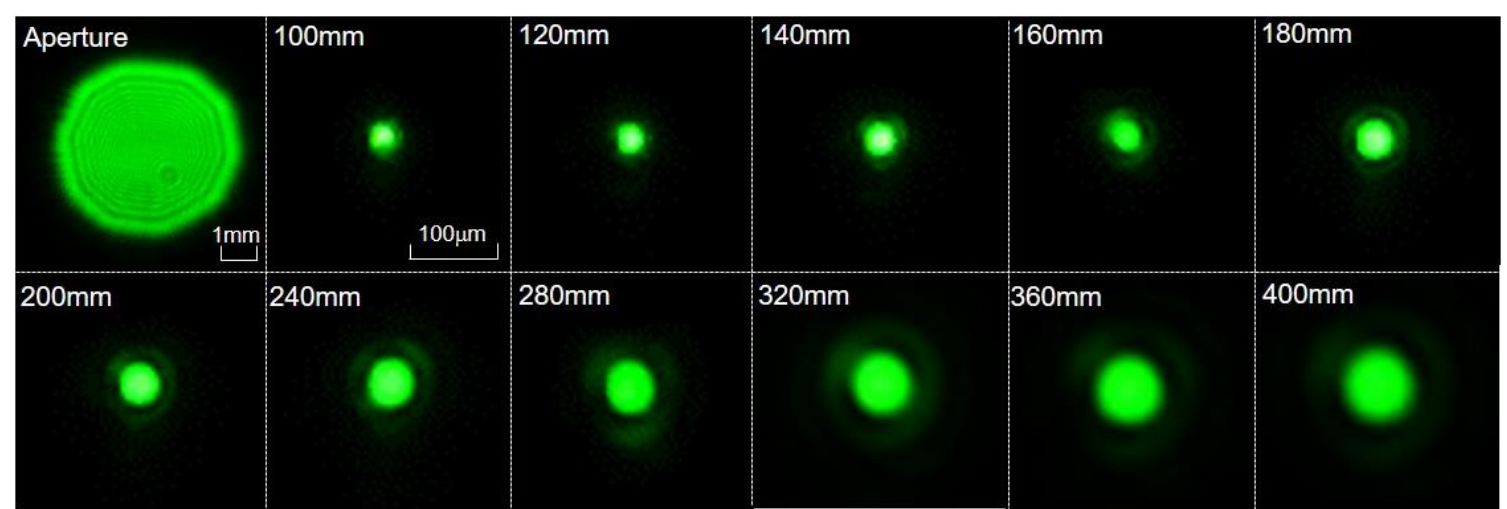

Figure 5. Experimentally recorded intensities of circular aperture and the focused PSFs with respect to different focal lengths of the Fresnel lens profile projected on the phase-only LCoS device.

The analysis of the PSF size and the intensity distribution are shown in Fig. 6. We can see that as the focal length increases, the PSF size increases as well, which follows a linear trend. The PSF size is $20.7 \mu \mathrm{m}$ at $100 \mathrm{~mm}$ and $45.58 \mu \mathrm{m}$ at $400 \mathrm{~mm}$. The experimental PSF size is larger than the theoretical value, and the different between them decreases as the focal length increases. This is due to the phase Fresnel lens, the phase changes more rapidly at the edge than at the center. However, the LCoS device has a fixed pixel size, so that it cannot sufficiently represent the rapid phase change at the edge of the phase Fresnel lens. This phenomenon is more dominant when we use a phase Fresnel lens with short focal length. Although the phase Fresnel lens comparably performs worse at short focal length, we can still see that the minimum PSF size is obtained at the shortest focal length. 




Figure 6. The full waist at half maximum (FWHM) of the spots at different focal lengths.

Once the relationship between the focal length and the PSF size is obtained, the focal length of the Fresnel lens is fixed as $200 \mathrm{~mm}$ for the remaining experiment. To laterally shift the PSFs, blaze gratings with different pitches have been applied onto the phase Fresnel lens. The laterally shifted PSFs are recorded and shown in Fig. 7. Each shift order represents an angle shift of $0.73^{\circ}$, at the focal length of $200 \mathrm{~mm}$, each lateral shift equals $1.28 \mathrm{~mm}$. Therefore, in the experiment, \pm 7 lateral shift order stands for $\pm 5.11^{\circ}$ or lateral shift of $8.96 \mathrm{~mm}$, which can support illumination angle up to $\pm 65^{\circ}$. Theoretically, for the LCoS device we use, the lateral shift can be up to $\pm 9.53^{\circ}$. From the results in Fig. 7 , we can see that the lateral shifted scanning spots can still maintain good profiles even at large angles. However, due to the insufficient representation of the blaze grating, the diffraction efficiency drops at large angles, which we can directly tell from the intensity drop of the higher orders, but the intensity variation doesn't affect the reconstruction process of the recorded hologram after normalization. Note that as we shift the targeted spot to higher angle, vague spots appear in the corner of the main spot, which is due to the double reflection on the ITO glass of the LCOS device. However, the intensity of the vague spots are much lower than the targeted main spot, hence it has very limited impact on the holographic recording process.

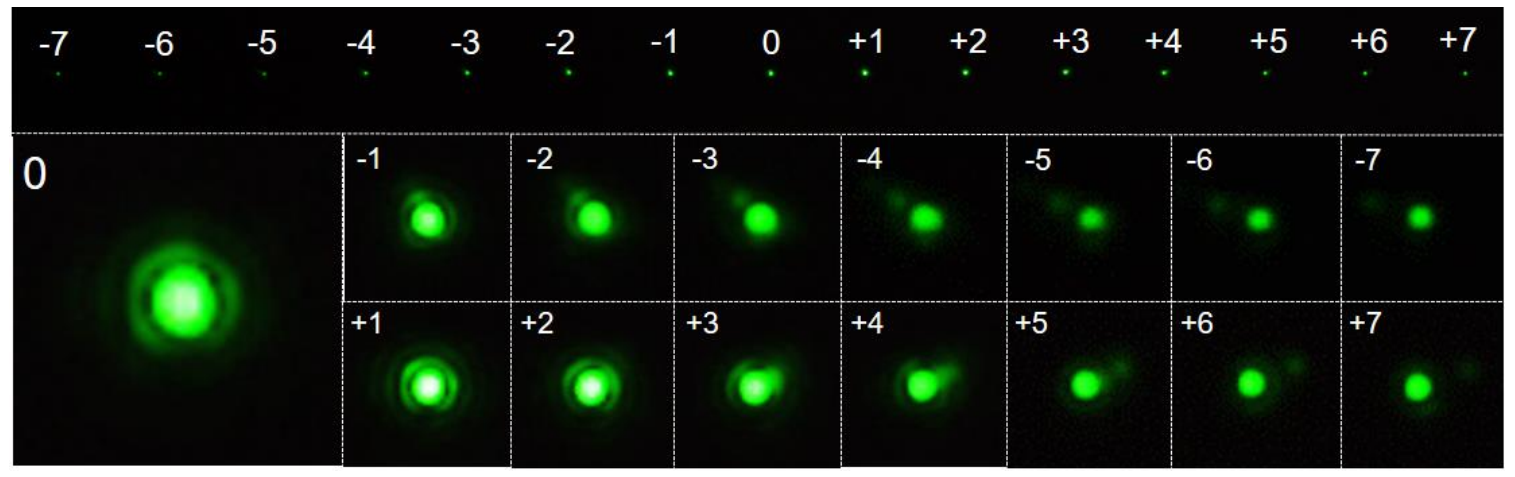

Figure 7. Laterally shifted scanning PSF $\mathrm{s}$ at the focal length of $200 \mathrm{~mm}$. The shift angle discrepancy is $0.73^{\circ}$. 


\subsection{SLM-based aberration-compensated SA-DHM}

In order to perform the aberation correction with the compact SLM-based beam steering SA-DHM, few calibration steps must be followed as shown in Fig. 8. For the wavefront aberration compensation process, different angles of plane wave illumination are experimentally recorded and then mapped with the Zernike polynomials aberration model to generate a conjugation wavefront. The conjugation wavefront is then sent to the phase modulation of LCoS device to iteratively compensate the aberration. The calibrated measurement procedure steps are briefly shown in Fig. 8.

The example of the ideal $\mathrm{CGH}$ and the aberration compensated $\mathrm{CGH}$ mentioned in step 1 and step 5 of the Fig. 8 are shown in Fig. 9(a) and 9(b) respectively. The sectional profiles

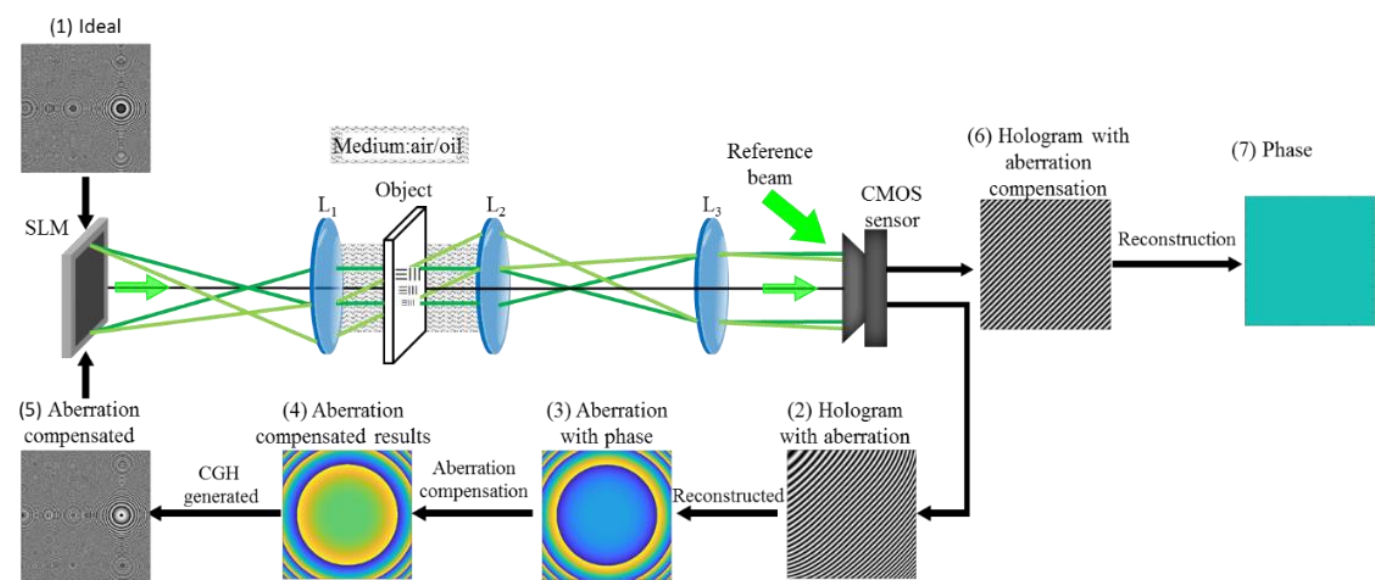

Figure 8. Calibration measurement procedure showing the steps to be followed to achieve the wavefront aberration-compensated imaging.

(a)



(b)

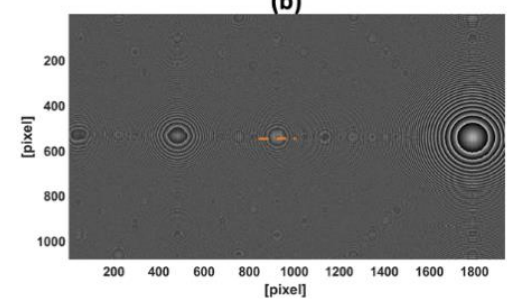

(c)

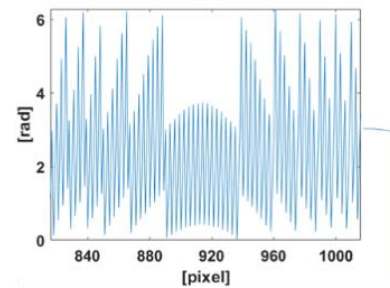

(d)

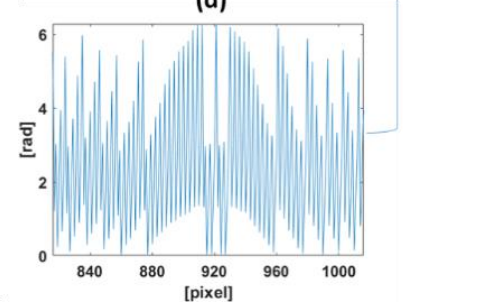

(e)

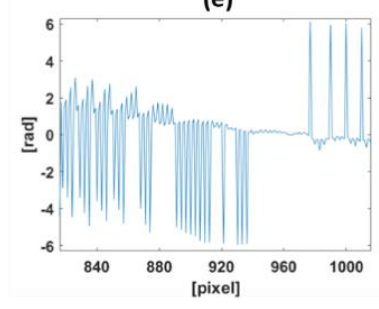

Figure 9. Designed CGH (a) ideal case, (b) for aberration correction; (c) and (d) are the corresponding sectional profiles in ROI of (a) and (b), respectively. The difference between (c) and (d) is the influence of aberrations in the CGH were shown in (e). 
of the dashed region of interest (ROI) of Fig 9(a) and 9(b) are shown in 9(c) and 9(d) respectively. The difference in the sectional profile are shown in Fig 9(e), which is due to the aberration influence in the $\mathrm{CGH}$.

The experimental setup of the compact SLM-based beam steering with aberrationcompensated SA-DHM are shown in Fig. 10, which adapts modified Mach-Zehnder interferometric architecture with phase-only SLM. A pair of 4-f telescopic imaging systems are used to illuminate and detect the object wave, in which the SLM is applied to substitute the rear lens in the systems. The first steering based illumination telescopic imaging lens $\left(S L M-M O_{1} ; f_{1}=200 \mathrm{~mm}, M O_{1}: N A=0.9,100 \times\right)$ is used to condense the plane wave to illuminate the sample. The resultant object wave affected by the aberration is collected by the detection 4- $f$ telescopic imaging lens $\left(f_{2}=200 \mathrm{~mm}, \mathrm{MO}_{2}: \mathrm{NA}=0.9,100 \times\right)$ in the intermediate image plane. The object wave is then interfered with the reference wave to generate a digital hologram on the CMOS image sensor. The accurate aberration implication and its correction are implemented and studied for two different cases such as without oil immersed microscope objective lens (case-1) and with oil immersed microscope objective lens (case-2) as shown in Fig. 10 (ii)-(a) and (ii)-(b), respectively.

For the aberration compensation process, the plane wave illumination at different scanning angle are experimentally recorded and then mapped with the Zernike polynomials aberration model to generate a conjugation wavefront. The conjugated wavefront is then sent to the SLM to iteratively compensate the aberration. The following sections will describe the experimental validation and analysis.

(i)

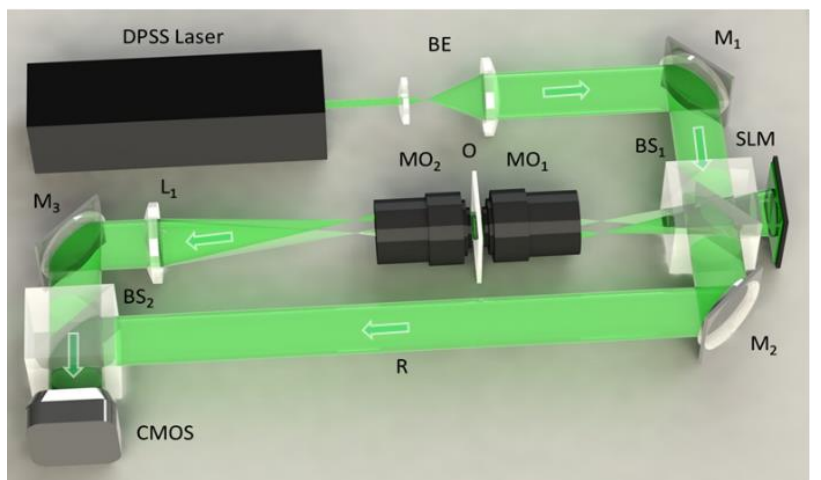

(ii)

(a)

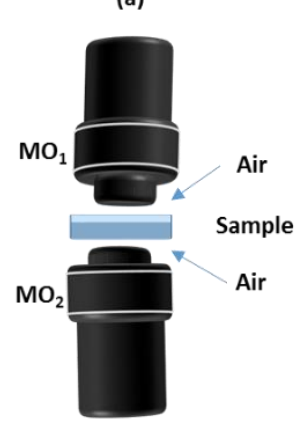

(b)

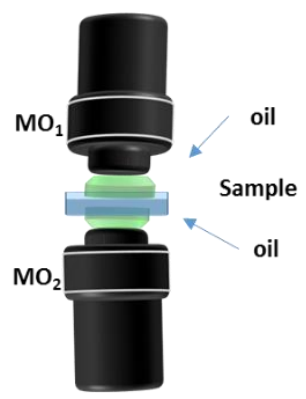

Figure 10. (i) Experimental schematic of compact beam steering with wavefront aberration compensated SA-DHM; (ii) illustration of without oil (a) and with oil (b) immersed microscope objective lens. 


\subsection{Experimental results and analysis}

\subsubsection{Aberration compensation validation}

To validate the proposed technique, the influence of aberration on the PSFs and reconstructed images are tested. For the accurate aberration compensation, two different cases without oil immersion microscope objective lens (case-1) and with oil immersed objective lens (case-2) are shown in Fig. 10 (ii)(a) and (ii)-(b) respectively. For both case- 1 and case- 2 , the normal incident $\left(0^{\circ}\right)$ and the inclined incident $\left(65^{\circ}\right)$ with and without aberration compensation (AC) are shown in Fig. 11(i):(a)-(d) and 11(ii):(a)-(d) respectively. The results in both case- 1 and case- 2 show a good agreement with the accurate aberration compensation. Further a quantitative analysis is conducted and the cross-sectional profiles along $x$-axis and $y$-axis for case- 1 and case- 2 are shown in Figures 11(i):(e)-(h) and 11(ii):(e)-(h) respectively. The results in Fig. 11 show that the phase distributions after aberration compensation at normal incident and inclined incident conditions can perform acceptable phase sensitivity on the reconstruction images.

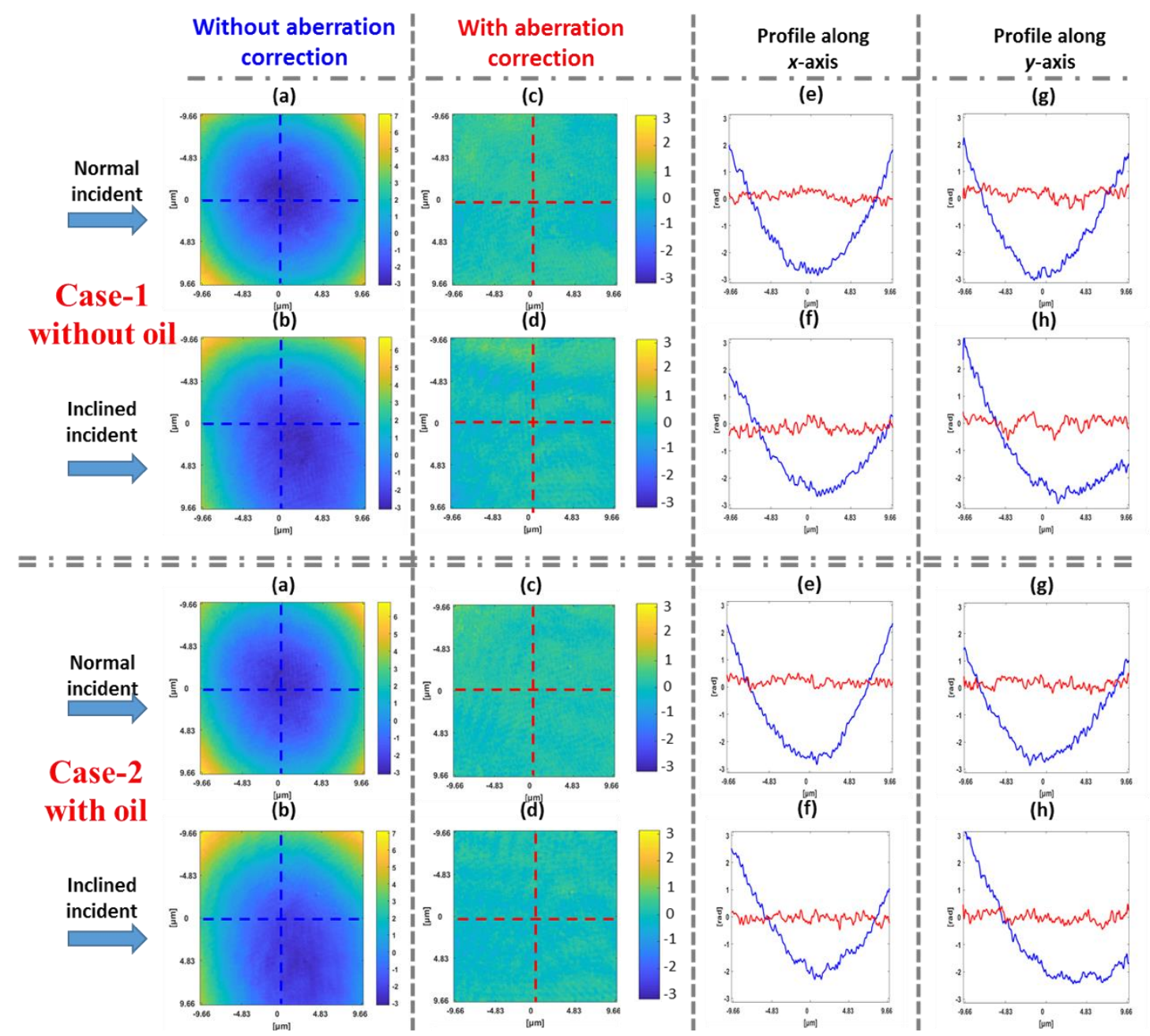

Figure 11. Reconstructed phase results: normal and inclined incidents correspond to without and with aberration compensation of two cases with its cross-sectional profiles along $x$ - and $y$ - axis. 


\subsubsection{Isotropic resolution enhancement}

In a conventional coherent imaging system, the theoretical resolution with normal aperture is approximately $455 \mathrm{~nm}$ for the wavelength of $532 \mathrm{~nm}$. This can be improved by SA method, SA-DHM can be resolved down to $227 \mathrm{~nm}(0.77 \lambda / 2 \mathrm{NA})$. In the proposed compact beam steering with aberration-compensated SA-DHM system, a standard spoken target having the minimal line width down to $150 \mathrm{~nm}$ (300 nm/pair) is used as sample for the validation. The experimental results of the reconstructed images of the spoken target corresponding to case-1 and case- 2 are shown in Fig. 12. For case-1 without oil immersed microscopic objective lens, the result for normal incident is shown in Fig. 12(i):(a), SA result without AC is shown in Fig. 12(i):(b) and SA result with AC is shown in Fig. 12(i):(c). Their cross-sectional profiles of circle marked ROI are shown in Fig. 12(i):(d), which shows that SA result with $A C$ can resolve some high frequency region of $150 \mathrm{~nm}$ but the sectional profile is not evenly distributed, which might be due to the perturbations created by the surrounding environment.



Figure 12. Experimental results: reconstructed image of the spoken target under without and with oil immersed microscope objective lens. The ROIs in the reconstructed images as marked by circled lines (a) normal aperture without aberration compensation, (b) synthetic aperture without aberration compensation in all directions, and (c) synthetic aperture with aberration compensation in all directions. (d) Comparison of the cross-sectional profiles of the line pairs amplitudes along the respected ROIs. 
For case-2 with oil immersed microscopic objective lens, the results are shown in a similar manner, and we can see that SA with AC can resolve high frequency region of $150 \mathrm{~nm}$ with good contrast. For more quantitative analysis of the isotropic resolution enhancement, the spatial frequency and the polar plot response of the spoken target corresponding to case- 1 and case- 2 are studied and the results are shown in Fig. 13. From the reconstructed spoken target results, the linearity behavior is analyzed for the two cases without $A C$ and with AC, which are shown in Fig. 13(a) and 13(b) respectively. Even though both the cases show improvement after AC in Fig. 13(b), but case-2 results show good linearity response compared to case-1.

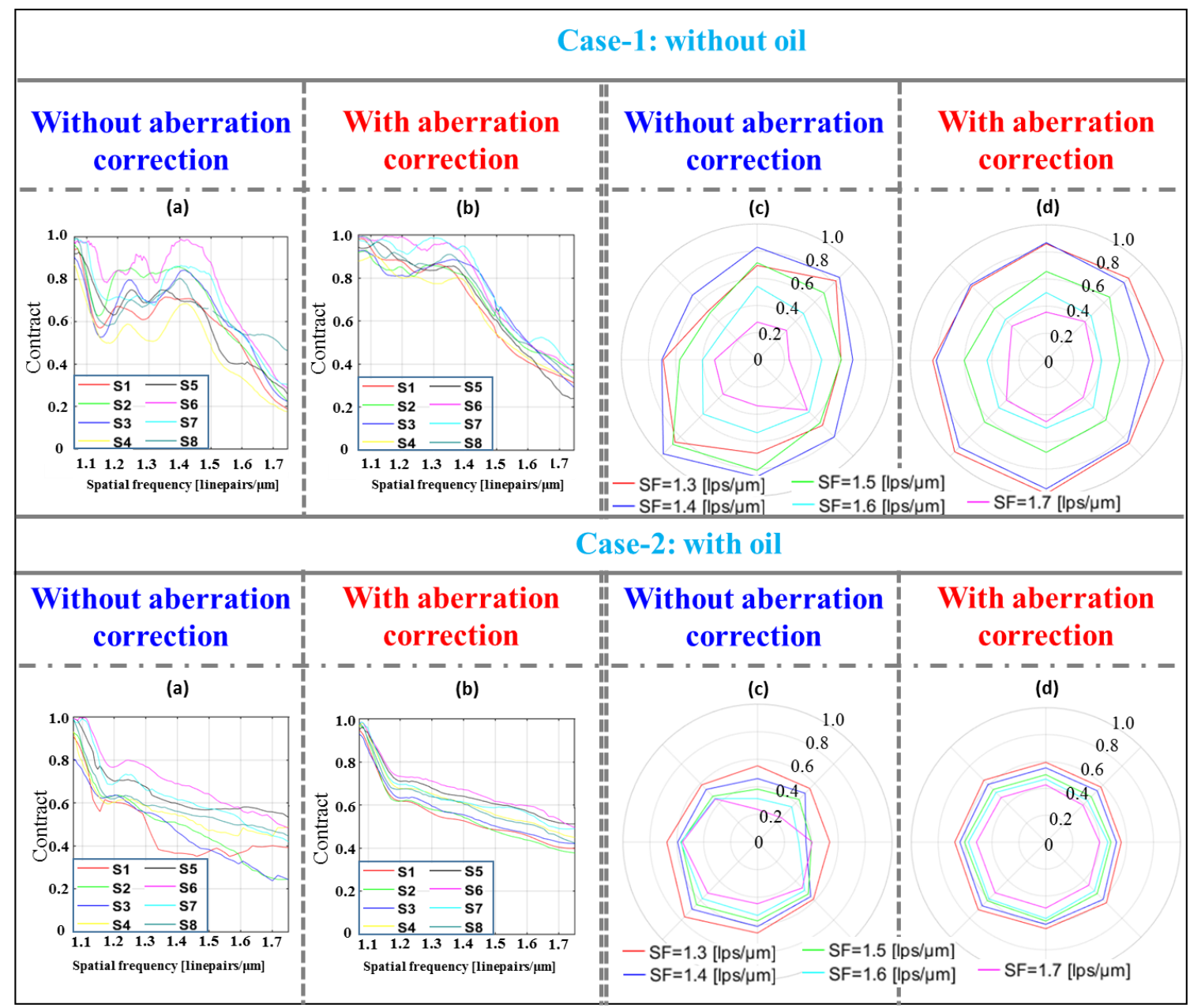

Figure 13. Spatial frequency and isotropically resolved profile analysis of experimentally reconstructed images of the spoken target. The SAs with and without aberration compensation have been divided into eight different segments with different spatial frequency in the reconstructed images. The amplitude contrasts of different segments for the two cases of SA applied without aberration compensation (a), and with aberration compensation are shown in (a) and (b) respectively. Similarly, polar plot analysis of both cases without $A C$ and with $A C$ are shown in (c) \& (d) respectively. 
Finally, the polar plot of each segment in the spoken target corresponding to the two cases are analyzed and plotted in Fig. 13(c) and 13(d) respectively. The AC results of case-1 show an improved profile but struggle to resolve the sample profile in isotropic manner. On the other side, case-2 results after aberration correction show an isotropically enhanced resolvable profile throughout the sample as shown in Fig. 14(d). The results show that accurate aberration correction will benefit both the cases.

\section{Conclusions}

This study has proposed and experimentally demonstrated a mechanical movementfree SLM-based compact beam steering technique with active aberration compensation for synthetic aperture digital holographic microscope. The optimization of the proposed compact beam steering system shows a good image quality, which has the potential to replace the traditional mechanical scanning mechanism with three significant advantages: free from mechanical noise, uniform illumination with aberrationcompensated scanning at different angles and more compact setup for system operation. An extensive analysis has been carried out on the aberration implications for cases without oil immersion and with oil immersed objective lens with the potential usage of the accurate aberration compensation technique. A spoke target has been used as a sample and the results show that the system has the capability of isotropic resolution enhancement in resolving $150 \mathrm{~nm}$ line width for the wavelength of $532 \mathrm{~nm}$. It is clear that the demonstrated method has the potential to be implemented in the real systems [40] for high-quality aberration-free 2D/3D imaging of bio-samples at its native culture level in everyday applications.

\section{Acknowledgements}

This work is financially supported in part by the UK Engineering and Physical Sciences Research Council (EPSRC) through the EPSRC Centre for Doctoral Training in Integrated Photonic and Electronic Systems (EP/L015455/1), the National Taiwan Normal University for Promoting International Collaboration \& Research Project, the Ministry of Science and Technology, Taiwan: MOST 108-2221-E-003 -019-MY3.

\section{Author's contribution}

CJC and DC conceived the idea and supervised the project. YD optimized the LCOS device and tested the beam steering architecture. $\mathrm{CH}, \mathrm{BV}$ and $\mathrm{XJ}$ conducted the experiments and data analysis. All the authors discussed the results and equally contributed to the manuscript. 


\section{References}

1. U. Schnars and W. Jueptner, Digital Holography (Springer, 2005).

2. M. K. Kim, Digital Holographic Microscopy: Principles, Techniques, and Applications (Springer New York, 2011).

3. W. Osten, A. Faridian, P. Gao, K. Körner, D. Naik, G. Pedrini, A. K. Singh, M. Takeda, and M. Wilke, Appl. Opt 53, G44 (2014).

4. YC Lin, C. J. Cheng, and L. C. Lin, "Tunable time-resolved tick-tock pulsed digital holographic microscopy for ultrafast events". Opt. Lett 42, 2082-2085 (2018).

5. J. H. Massig, "Digital off-axis holography with a synthetic aperture," Opt. Lett 27, 2179-2181 (2002).

6. M. K. Kim, Digital Holographic Microscopy: Principles, Techniques, and Applications (Springer New York, 2011).

7. P. Ferraro, A. Wax, and Z. Zalevsky, Coherent Light Microscopy: Imaging and Quantitative Phase Analysis (Springer, 2011).

8. Y. Cotte, F. Toy, P. Jourdain, N. Pavillon, D. Boss, P. Magistretti, P. Marquet, and C. Depeursinge, "Marker-free phase nanoscopy," Nat. Photonics 7, 113-117 (2013).

9. Y. L. Lee, Y. C. Lin, H. Y. Tu, and C. J. Cheng, "Phase measurement accuracy in digital holographic microscopy using a wavelength-stabilized laser diode," J. Opt 15, 025403 (2013).

10. V. R. Besaga, A. V. Saetchnikov, N. C. Gerhardt, A. Ostendorf, and M. R. Hofmann, "Digital holographic microscopy for sub-um scale high aspect ratio structures in transparent materials," Opt Laser Eng 121, 441-447 (2019).

11. W. Luo, A. Greenbaum, Y. Zhang, and A. Ozcan, "Synthetic aperture based on-chip microscopy," Light Sci Appl 4, e261 (2015).

12. M. Kim, Y. Choi, C. F. Yen, Y. Sung, R. R. Dasari, M. S. Feld, and W. Choi, "High-speed synthetic aperture microscopy for live cell imaging," Opt. Lett 36, 148-150 (2011).

13. X. J. Lai, H. Y. Tu, C. H. Wu, Y. C. Lin, and C. J. Cheng, "Resolution enhancement of spectrum normalization in synthetic aperture digital holographic microscopy," Appl. Opt 54, A51-A58 (2015).

14. H. Y. Tu, W. J. Hsiao, X. J. Lai, Y. C. Lin, and C. J. Cheng, "Synthetic aperture common-path digital holographic microscopy with spiral phase filter," J. Opt 19, 065604 (2017).

15. X. J. Lai, C. J. Cheng, Y. C Lin, and H. Y Tu, "Angular-polarization multiplexing with spatial light modulators for resolution enhancement in digital holographic microscopy", J. Opt 19,055607 (2017).

16. A. Stadelmaier and J. H. Massig, "Compensation of lens aberrations in digital holography," Opt. Lett 25, 1630-1632 (2000).

17. T. Colomb, J. Kühn, F. Charrière, C. Depeursinge, P. Marquet, and N. Aspert, "Total aberrations compensation in digital holographic microscopy with a reference conjugated hologram," Opt. Exp 14, 4300-4306 (2006).

18. T. Colomb, E. Cuche, F. Charrière, J. Kühn, N. Aspert, F. Montfort, P. Marquet, and C. Depeursinge "Automatic procedure for aberration compensation in digital holographic microscopy and applications to specimen shape compensation," Appl. Opt 45, 851-863 (2006).

19. F. Montfort, F. Charrière, T. Colomb, E, Cuche, P, Marquet, and C. Depeursinge "Purely numerical compensation for microscope objective phase curvature in digital holographic microscopy: influence of digital phase mask position," J. Opt. Soc. Am. A 23, 2944-2953 (2006).

20. T. Colomb, F. Montfort, J. Kühn, N. Aspert, E. Cuche, A. Marian, F. Charrière, S. Bourquin, P. Marquet, and C. Depeursinge, "Numerical parametric lens for shifting, magnification, and complete aberration compensation in digital holographic microscopy," J. Opt. Soc. Am. A 23, 3177-3190 (2006).

21. W. J. Zhou, Y. J. Yu, and A. Asundi, "Study on aberration suppressing methods in digital microholography," Opt Laser Eng 47, 264-270 (2009).

22. K. W. Seo, Y. S. Choi, E. S. Seo, and S. J. Lee, "Aberration compensation for objective phase curvature in phase holographic microscopy," Opt. Lett 37, 4976-4978 (2012). 
23. C. Zuo, Q. Chen, W. Qu, and A. Asundi "Phase aberration compensation in digital holographic microscopy based on principal component analysis," Opt. Lett 38, 1724-1726 (2013).

24. D. N. Deng, J. Z. Peng, W. J. Qu, Y. Wu, X. L. Liu, W. Q. He, and X. Peng, "Simple and flexible phase compensation for digital holographic microscopy with electrically tunable lens," Appl. Opt 56, 60076014 (2017).

25. I. Y. Choi, K. R. Lee, and Y. K. Park, "Compensation of aberration in quantitative phase imaging using lateral shifting and spiral phase integration," Opt. Exp 25, 30771-30779 (2017).

26. H. Liu, J. Bailleul, B. Simon, M. Debailleul, B. Colicchio, and O. Haeberlé, "Tomographic diffractive microscopy and multiview profilometry with flexible aberration correction," Appl. Opt 53, 748-755 (2014).

27. C. Maurer, A. Jesacher, S. Bernet, and M. R. Marte, "What spatial light modulators can do for optical microscopy," Laser Photon Rev 5, 81-101 (2011).

28. M. Booth, D. Andrade, D. Burke, B. Patton, and M. Zurauskas, "Aberrations and adaptive optics in super-resolution microscopy," Microscopy 64, 251-261 (2015).

29. A. Kus, W. Krauze, and M. Kujawińska, "Active limited-angle tomographic phase microscope". J. Biomed. Opt 20, 111216 (2015).

30. B. Vinoth, H.Y. Tu, X. J. Lai and C. J, Cheng, "Adaptive wavefront correction structured illumination holographic tomography". Sci Rep 9, 10489 (2019).

31. X. J. Lai, H. Y. Tu, Y.C. Lin, and C. J. Cheng, "Coded aperture structured illumination digital holographic microscopy for superresolution imaging". Opt. Lett 43, 1143-1146 (2018)

32. G. D. Love, "Wave-front correction and production of Zernike modes with a liquid-crystal spatial light modulator," Appl. Opt 36, 1517-1524 (1997).

33. G. Lazarev, A. Hermerschmidt, S. Krüger, and S. Osten, "LCoS spatial light modulators: Trends and applications," Wiley-VCH, 1-30 (2012).

34. R. Horstmeyer, R. Heintzmann, G. Popescu, L. Waller and C. H. Yang, "Standardizing the resolution claims for coherent microscopy," Nat. Photonics 10, 68-71 (2016).

35. A. O. Yontem, and L. Onural. "Integral imaging using phase-only LCoS spatial light modulators as Fresnel lenslet arrays," JOSA A, 28.11:2359-2375 (2011).

36. D. Wang, et al, "High-fidelity numerical realization of multiple-step Fresnel propagation for the reconstruction of digital holograms," Appl. Opt 47, D12-D20 (2008).

37. Y. Deng, and D. Chu. "Effect of masking phase-only holograms on the quality of reconstructed images," App. Opt 55, 3158-3164 (2016).

38. Y. Deng, and D. Chu. "Filling factor characteristics of masking phase-only hologram on the quality of reconstructed images." Proc.SPIE. 9771, Practical Holography XXX: Material and Applications, 97710M (2016).

39. Z. Zhang, H. Yan, B. Roberston, M. Redmond, M. Pivnenko, N. Collings, W.A.Crossland and D. Chu, "Diffraction abased phase compensation method for phase-only liquid crystal on silicon devices in operation," Appl. Opt 51, 3837-3846 (2012).

40. B. Vinoth, X. J. Lai, Y. C. Lin, H. Y. Tu, and C. J. Cheng, "Integrated dual-tomography for refractive index analysis of free-floating single living cell with isotropic superresolution”. Sci. Rep 8, 5943 (2018). 